\title{
A special issue on calcium dynamics of the heart: remodeling of ion channels and regulatory pathways
}

\author{
Dan J. Bare ${ }^{1} \cdot$ Lixia Yue $^{2} \cdot$ Xun $^{\mathrm{Ai}^{1}}{ }^{1}$ \\ Received: 27 January 2021 / Revised: 27 January 2021 / Accepted: 1 February 2021 / Published online: 5 March 2021 \\ (C) The Author(s), under exclusive license to Springer-Verlag GmbH, DE part of Springer Nature 2021
}

Calcium $\left(\mathrm{Ca}^{2+}\right)$ has been identified as an essential regulator of cardiac function since 1883 [16]. To meet the ever-changing metabolic and energy demands placed on the heart by the body, there is a strictly synchronized control of coupling between excitation and contraction, which is defined by sequential dynamics of $\mathrm{Ca}^{2+}$ signaling that links the cell membrane depolarization, ion channel activation, and the events of $\mathrm{Ca}^{2+}$-induced- $\mathrm{Ca}^{2+}$-release from the sarcoplasmic reticulum $\mathrm{Ca}^{2+}$ store to the activation of the myofibrillar contractile machinery during each heartbeat. A balanced intracellular $\mathrm{Ca}^{2+}$ homeostasis is maintained by highly coordinated actions of the intracellular $\mathrm{Ca}^{2+}$ cycling machinery including $\mathrm{Ca}^{2+}$ channels, $\mathrm{Ca}^{2+}$ pumps/transporters located on the plasma membrane, endo/sarcoplasmic reticulum (ER/SR) and mitochondrion in myocytes and fibroblasts $[1,4,9]$. In this Special Issue, we compiled a coherent set of review and original research articles to introduce the major advances regarding our current understanding of the cardiac $\mathrm{Ca}^{2+}$ signaling via ion channels including voltage-gated $\mathrm{Ca}^{2+}$ channels, ligand-gated $\mathrm{Ca}^{2+}$ channels, ryanodine receptors (RyR2s), inositol 1,4,5-trisphosphate receptors $\left(\mathrm{IP}_{3} \mathrm{Rs}\right), \mathrm{Ca}^{2+}$ pumps/transporters, and transient receptor potential (TRP) channels (Fig. 1) under both physiological and pathological conditions. Moreover, translational studies aimed at developing novel therapeutic strategies to prevent and treat patients with heart failure and cardiac arrhythmias were also discussed.

This article is part of the special issue on Calcium Signal Dynamics in Cardiac Myocytes and Fibroblasts: Mechanisms in Pflügers Archiv_European Journal of Physiology

Lixia Yue

lyue@uchc.edu

$\triangle$ Xun Ai

Xun_Ai@rush.edu

1 Department of Physiology and Biophysics, Rush University Medical Center, Chicago, IL 60612, USA

2 Department of Cell Biology, Calhoun Cardiology Center, University of Connecticut Health Center, Farmington, CT 06030, USA
In this Special Issue, several articles (Blatter et al., Lahiri et al., McKee et al., Hamilton et al., Wang et al., Xie et al., Rosenberg et al., Fill et al.) $[2,7,8,12,13,17-19]$ have thoroughly reviewed the physiological role of the excitation-contraction coupling (ECC) in the cardiomyocyte and a well-established concept that defective intracellular $\mathrm{Ca}^{2+}$ cycling acts as a key contributing factor to an elevated predisposition for arrhythmias and/or impaired cardiac contractility in pathologically altered hearts. Under certain conditions such as exercise, aging, excessive alcohol exposure, obesity, diabetes, inherited mutational burdens, ischemia, systemic inflammation, these extrinsic/intrinsic stress stimuli trigger adaptive remodeling of $\mathrm{Ca}^{2+}$ handling machinery and regulatory signaling pathways in cardiac cells that are necessary to deliver an adequate acute response but also ensures long-term survival. Unfortunately, sustained stimulation of these stress stimuli ultimately disrupts the normal dynamics of cardiac $\mathrm{Ca}^{2+}$ handling, which results in myocardial maladaptive remodeling toward cardiac dysfunction. In addition, disrupted intracellular $\mathrm{Ca}^{2+}$ cycling and SR $\mathrm{Ca}^{2+}$ mishandling critically contribute to the development of fatal ventricular fibrillation (a major cause of sudden cardiac death) and atrial fibrillation (the most common arrhythmia, with a higher mortality due to significantly increased risk for stroke and heart failure). Moreover, detailed discussions by Lahiri et al., McKee et al., Hamilton et al., Wang et al., Rosenberg et al., $[8,12,13,17,18]$ were also included regarding the new findings of regulatory mechanisms of stress stimuli (i.e., aging, excessive alcohol abuse, inflammation, ischemic-reperfusion) or inherited gene mutations (i.e., Catecholaminergic Polymorphic Ventricular Tachycardia (CPVT) and Long QT Syndrome (LQTS)) in disrupted SR $\mathrm{Ca}^{2+}$ dynamics via altered channel function of RyR2, $I_{3} R 2$, and STIM1-Orail, which lead to changed $\mathrm{SR} \mathrm{Ca}^{2+}$ load and significantly increased diastolic SR $\mathrm{Ca}^{2+}$ leak promoting the initiation or progression of arrhythmias and heart failure. Altered enzyme activity and/or changed interactions between kinases/protein phosphatases and ion channels critically contribute to these stress-evoked changes in the $\mathrm{Ca}^{2+}$ homeostasis and cardiac electrical and mechanical dysfunction. While hyperactivation of CaMKII $\delta$ is well-known to promote 


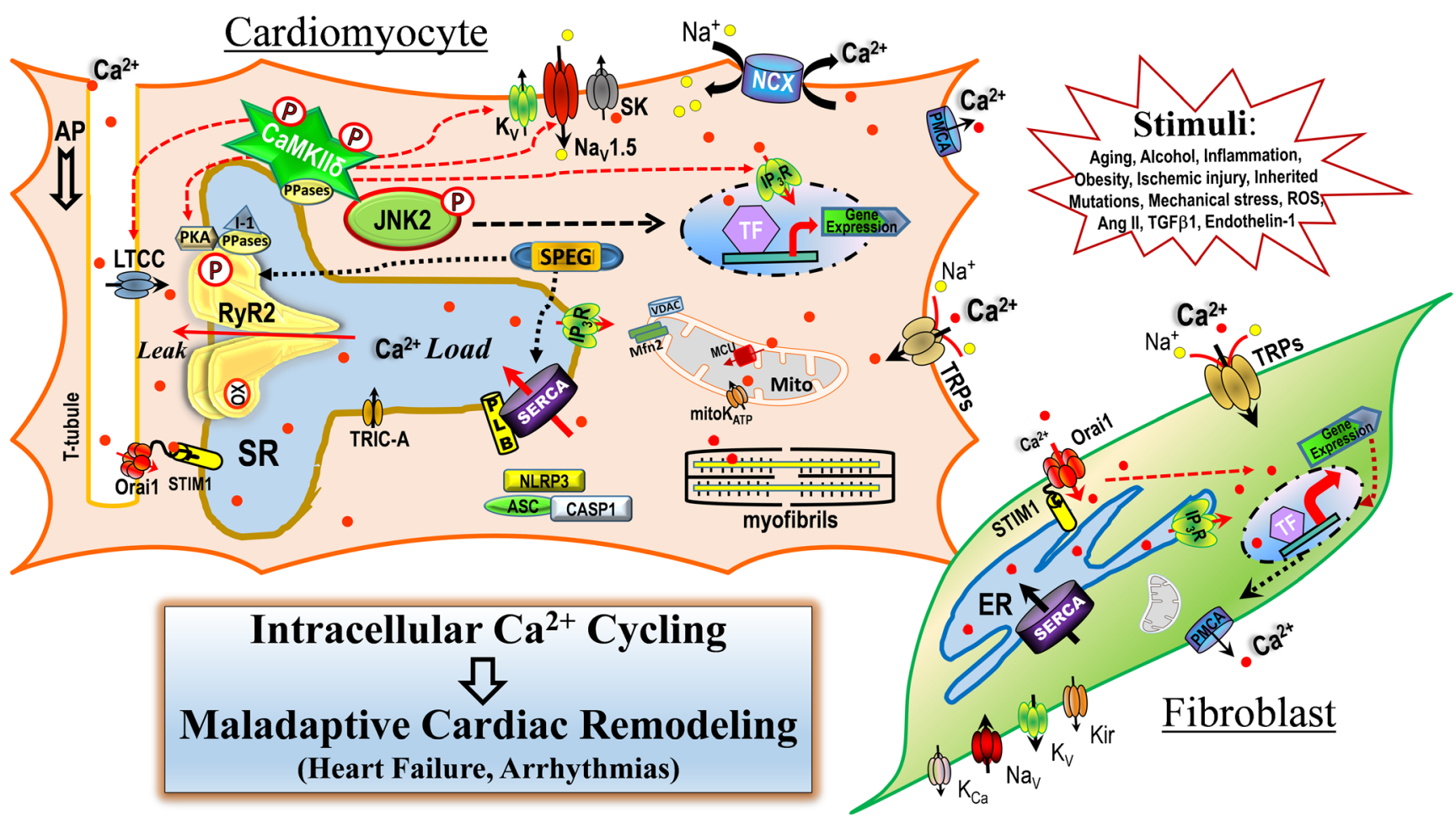

Fig. 1 Schematic diagram of demonstrated mechanisms of intracellular $\mathrm{Ca}^{2+}$ cycling-mediated cardiac maladaptive remodeling which could lead to various types of cardiac functional abnormalities such as heart failure and arrhythmias

maladaptive cardiac remodeling during disease onset and progression, recent studies have uncovered crucial roles of several important kinases and protein phosphatases including stressresponse kinase JNK2, striated muscle preferentially expressed protein kinase (SPEG), protein phosphatases PP1, PP2A, and endogenous PP1 inhibitor protein (I-1) in intracellular $\mathrm{Ca}^{2+}$ cycling and $\mathrm{SR} \mathrm{Ca}^{2+}$ dynamics as well as enhanced arrhythmogenicity in the stressed heart. These newly identified channel modifiers within the macro-molecular complex of the $\mathrm{Ca}^{2+}$ handling machinery suggest the complexity of the regulation of the ion channel activities and intercellular $\mathrm{Ca}^{2+}$ homeostasis in cardiac myocytes.

The beat-to-beat $\mathrm{Ca}^{2+}$ signaling event occurs in both atrial and ventricular cells but with some notable functionally relevant and structurally based differences. Blatter et al. [2] provide a timely review regarding the distinctive mechanisms of ECC in the atria and ventricles, while the functional role of an "auxiliary" pathway of $\mathrm{Ca}^{2+}$ release involving the less studied cardiac $\mathrm{IP}_{3} \mathrm{R}$ type $2\left(\mathrm{InsP}_{3} \mathrm{R}-2\right) \mathrm{Ca}^{2+}$ release channel for atrial $\mathrm{ECC}$ and atrial alternans in arrhythmogenesis were also presented. Moreover, accumulating studies suggest that mitochondrial $\mathrm{Ca}^{2+}$ and the mitochondrial $\mathrm{Ca}^{2+}$ handling units such as mitochondrial $\mathrm{Ca}^{2+}$ uniporters (MCU) are also involved in physiological $\mathrm{Ca}^{2+}$ homeostasis as well as pathological intracellular $\mathrm{Ca}^{2+}$ mishandling in diseased hearts. Ernst et al. [5] reported a new approach to assess the coupling between mitochondrial $\mathrm{Ca}^{2+}$ and cytosolic $\mathrm{Ca}^{2+}$ dynamics using genetically-encoded $\mathrm{Ca}^{2+}$ indicators (GECIs) in human- induced pluripotent stem cell-derived cardiomyocytes (hiPSC-CMs) and adult cardiomyocytes (ACMs). It should be noted that cardiac maladaptive function during the process of pathological myocardial remodeling (i.e., HF, AF) is well known to result in structural and biochemical changes related to the complex alterations of ion channel function, protein abundance, and protein post-translational modifications. Biochemical assays and proteomic analyses have thus been commonly employed. Rennison et al. [15] reported studies using a proteomic approach on the left atrial appendage from AF patients undergoing mitral valve repair and Maze surgery, showing significant expression changes in proteins related to mitochondrial energy production, which could contribute to cardiac contraction and electrophysiological remodeling in promoting AF pathogenesis. In addition, Yang et al. [20] also reported alterations of several housekeeping proteins in human heart tissue that were heart chamber-specific and highly disease context dependent in their expression levels. Past genetic studies have suggested that a strong heritable component is associated with the risk of AF and is inclusive of genes related to transcription factors. Due to the complexity of the etiology and progression of cardiac diseases, contributions of expression of genes and proteins in pathological $\mathrm{Ca}^{2+}$ mishandling and cardiac dysfunction are clearly worthy of future investigation. A much better understanding of the complex genetic regulatory networks involved in the cardiac pathogenesis will be critical for developing novel personalized therapies for patients. 
In addition to the critical regulation of ECC, excitationtranscriptional coupling, and various other intracellular signaling cascades in cardiac contractile dysfunction and cardiac arrhythmia development, other ion channels including $\mathrm{Ca}^{2+}$ activated $\mathrm{K}^{+}$channels and TRP channels also contribute to the intracellular $\mathrm{Ca}^{2+}$ homeostasis. $\mathrm{Ca}^{2+}$-activated small conductance $\mathrm{K}^{+}$channels, SK, or apamin-sensitive $\mathrm{SK}\left(\mathrm{I}_{\mathrm{KAS}}\right)$ are differentially expressed in the heart. Zhang et al. [21] presented an excellent review covering the discovery of SK channels in the heart to the most recent update in the understanding of the roles of SK channels in arrhythmias and HF. Chen et al. [3] concisely summarized how $\mathrm{I}_{\mathrm{KAS}}$ channels are regulated and are involved in arrhythmias and proposed that sexual dimorphism in autonomic control of $\mathrm{I}_{\mathrm{KAS}}$ plays a role in $J$ wave syndrome. In contrast to the SK channel, the $\mathrm{Ca}^{2+}$-activated TRPM isoform 4 (TRPM4) is a non-selective monovalent cation channel impermeable to $\mathrm{Ca}^{2+}$ ions. Dysfunction of TRPM4 contributions to electrophysiological abnormalities under a variety of inherited cardiac conduction block conditions and Brugada syndrome and is also associated with various forms of acquired heart defects such as arrhythmias and heart failure. $\mathrm{Hu}$ et al. [10] elegantly demonstrated how TRPM4 contributes to early afterdepolarization (EAD) and promotes arrhythmogenicity via CaMKII-dependent abnormal $\mathrm{Ca}^{2+}$ homeostasis. Feng et al. [6] provided new evidence suggesting that TRPM4 significantly upregulated in cardiac fibroblasts in heart failure patients; however its contribution in enhanced arrhythmogenicity in heart failure requires further investigation. Medert et al. [14] knocked down TRPM4 in adult hearts by using a myocyte-targeted AAV9-RNAi approach, providing a nice tool for future investigation regarding the functional role of TRPM4 in the heart. Whereas the ionic homeostasis of cardiac cells is finely tuned by multiple ion channels, transporters, and ionic exchangers in the plasma membrane, the ionic homeostasis of intracellular organelles is also delicately maintained by influx and efflux of different ions. Although it remains debatable in this research field, the potential contribution of the TRIC-A channel in mediating $\mathrm{K}^{+}$ influx to ER/SR to counter-balance $\mathrm{Ca}^{2+}$ release was thoroughly reviewed by Zhou et al. [22]. Not only is the ionic homeostasis in cardiac cells essential for normal heart function, but abnormal extracellular ionic concentrations resulting from pathological conditions such as ischemia and renal failure could also promote arrhythmias, which indicates the need for further investigation. In addition, King et al. [11] nicely demonstrated how the combined effects of $\mathrm{Na}^{+}$and $\mathrm{Ca}^{2+}$ can differentially modulate conduction during hyperkalemia and how enhancing determinants of ephaptic coupling may attenuate conduction changes in various physiologic conditions.

Over the past few decades, significant progress has been made in understanding the underlying mechanisms by which intracellular $\mathrm{Ca}^{2+}$ cycling mediates various types of cardiac functional abnormalities such has heart failure and arrhythmias. This
Special Issue highlights some of the major progresses that have been made regarding changes of intracellular $\mathrm{Ca}^{2+}$ homeostasis via ion channels regulated by either $\mathrm{Ca}^{2+}$-induced $\mathrm{SR} \mathrm{Ca}^{2+}$ release or a number of ion channel modifiers including kinases and protein phosphatases in response to various intrinsic or extrinsic stimuli. All of these exciting new perspectives lay the groundwork for future investigation such as the inter-relationship between kinases and protein phosphatases as well as between organelles in calcium handling and homeostasis in both normal function and in the pathological state. The complexity of the genetic regulatory networks in the dysfunction of intracellular cycling and maladaptive cardiac remodeling and disease onset and progression are also worthy of further investigation. Moreover, the interactions between SR, mitochondrion, and nucleus in orchestrating normal intracellular $\mathrm{Ca}^{2+}$ homeostasis as well as aberrant $\mathrm{Ca}^{2+}$ handling under pathological conditions are an interesting research direction deserving more attention. While the functional role of fibroblasts in cardiac electrophysiology and structural remodeling has recently emerged as a research point of interest, the interaction between myocytes and fibroblasts is clearly another new research direction in need of attention. These novel discoveries and new directions in future research will hopefully lead to targeted and effective therapies to prevent the maladaptive remodeling and disease progression in the heart.

Acknowledgements We thank motifolio.com for providing technical support to our graphical work.

Funding This work was supported by National Institutes of Health (P01HL06426, R01-AA024769, R01-HL113640, R01-HL146744, and AA024769S2 to XA; and R01-HL143750 to LY), and American Heart Association (19TPA34890022 to LY).

\section{Declarations}

Conflict of interest The authors declare that they have no conflict of interest.

Open Access This article is licensed under a Creative Commons Attribution 4.0 International License, which permits use, sharing, adaptation, distribution and reproduction in any medium or format, as long as you give appropriate credit to the original author(s) and the source, provide a link to the Creative Commons licence, and indicate if changes were made. The images or other third party material in this article are included in the article's Creative Commons licence, unless indicated otherwise in a credit line to the material. If material is not included in the article's Creative Commons licence and your intended use is not permitted by statutory regulation or exceeds the permitted use, you will need to obtain permission directly from the copyright holder. To view a copy of this licence, visit http://creativecommons.org/licenses/by/4.0/.

\section{References}

1. Bers DM (2002) Cardiac excitation-contraction coupling. Nature 415:198-205. https://doi.org/10.1038/415198a 
2. Blatter LA, Kanaporis G, Martinez-Hernandez E, OropezaAlmazan Y, Banach K (2021) Excitation-contraction coupling and calcium release in atrial muscle. Pflugers Arch. https://doi. org/10.1007/s00424-020-02506-X

3. Chen M, Fei Y, Chen TZ, Li YG, Chen PS (2021) The regulation of the small-conductance calcium-activated potassium current and the mechanisms of sex dimorphism in $\mathrm{J}$ wave syndrome. Pflugers Arch. https://doi.org/10.1007/s00424-020-02500-3

4. Eisner DA, Caldwell JL, Kistamás K, Trafford AW (2017) Calcium and excitation-contraction coupling in the heart. Circ Res 121:181195. https://doi.org/10.1161/circresaha.117.310230

5. Ernst P, Chen K, Tang Y, Kim S, Guan J, He J, Xie M, Zhang JJ, Liu XM, Zhou L (2021) Investigation into the difference in mitochondrial-cytosolic calcium coupling between adult cardiomyocyte and hiPSC-CM using a novel multifunctional genetic probe. Pflugers Arch. https://doi.org/10.1007/s00424-021-02524-3

6. Feng J, Zong P, Yan J, Yue Z, Li X, Smith C, Ai X, Yue L (2021) Up-regulation of transient receptor potential melastatin 4 (TRPM4) in ventricular fibroblasts from human failing heart. Pflugers Arch. https://doi.org/10.1007/s00424-021-02525-2

7. Fill M, Gillespie D (2021) Simulating cardiac Ca2+ release units: Effects of RyR cluster size and $\mathrm{Ca} 2+$ buffers on diastolic $\mathrm{Ca} 2+$ leak. Pflugers Arch. https://doi.org/10.1007/s00424-021-02539-w

8. Hamilton S, Veress R, Belevych A, Terentyev D (2021) The role of calcium homeostasis remodeling in inherited cardiac arrhythmia syndromes. Pflugers Arch. https://doi.org/10.1007/s00424-02002505-y

9. Hof T, Chaigne S, Recalde A, Salle L, Brette F, Guinamard R (2019) Transient receptor potential channels in cardiac health and disease. Nat Rev Cardiol 16:344-360. https://doi.org/10.1038/ s41569-018-0145-2

10. Hu Y, Kaschitza DR, Essers M, Arullampalam P, Fujita T, Abriel $\mathrm{H}$, Inoue R (2021) Pathological activation of CaMKII induces arrhythmogenicity through TRPM4 overactivation. Pflugers Arch. https://doi.org/10.1007/s00424-020-02507-w

11. King DR, ME II, Blair GA, Crandell I, Hanlon AL, Lin J, Hoeker G, Poelzing S (2021) The conduction velocity-potassium relationship in the heart is modulated by sodium and calcium. Pflugers Arch. https://doi.org/10.1007/s00424-021-02537-y

12. Lahiri SK, Aguilar-Sanchez Y, Wehrens XHT (2021) Mechanisms underlying pathological $\mathrm{Ca}(2+)$ handling in diseases of the heart. Pflugers Arch. https://doi.org/10.1007/s00424-020-02504-z
13. McKee C, Bare DJ, Ai X (2021) Stress driven cardiac calcium mishandling via a kinase-to-kinase crosstalk. Pflugers Arch. https://doi.org/10.1007/s00424-021-02533-2

14. Medert R, Jungmann A, Hildebrand S, Busch M, Grimm D, Flockerzi V, Müller OJ, Most P, Schumacher D, Freichel M (2021) Development of an AAV9-RNAi mediated silencing strategy to abrogate TRPM4 expression in the adult heart. Pflugers Arch. https://doi.org/10.1007/s00424-021-02521-6

15. Rennison JH, Li L, Lin CR, Lovano BS, Castel L, Wass SY, Cantlay CC, McHale M, Gillinov AM, Mehra R, Willard BB, Smith JD, Chung MK, Barnard J, Van Wagoner DR (2021) Atrial fibrillation rhythm is associated with marked changes in metabolic and myofibrillar protein expression in left atrial appendage. Pflugers Arch. https://doi.org/10.1007/s00424-021-02514-5

16. Ringer S (1883) A further contribution regarding the influence of the different constituents of the blood on the contraction of the heart. J Physiol 4(29-42):23-42. https://doi.org/10.1113/jphysiol. 1883.sp000120

17. Rosenberg P, Zhang H, Graham-Bryson V, Wang C (2021) SOCE in the cardiomyocyte: the secret is in the chambers. Pflugers Arch. https://doi.org/10.1007/s00424-021-02540-3

18. Wang X, Chen X, Dobrev D, Li N (2021) The crosstalk between cardiomyocyte calcium and inflammasome signaling pathways in atrial fibrillation. Pflugers Arch. https://doi.org/10.1007/s00424021-02515-4

19. Xie LH, Gwathmey JK, Zhao Z (2021) Cardiac adaptation and cardioprotection against arrhythmias and ischemia-reperfusion injury in mammalian hibernators. Pflugers Arch. https://doi.org/10. 1007/s00424-020-02511-0

20. Yang M, Yan J, Wu A, Zhao W, Qin J, Pogwizd SM, Wu X, Yuan S, Ai X (2021) Alterations of housekeeping proteins in human aged and diseased hearts. Pflugers Arch. https://doi.org/10.1007/s00424021-02538-x

21. Zhang X-D, Thai PN, Lieu DK, Chiamvimonvat N (2021) Cardiac small-conductance calcium-activated potassium channels in health and disease. Pflugers Arch. https://doi.org/10.1007/s00424-02102535-0

22. Zhou X, Li A, Lin PH, Zhou J, Ma J (2021) TRIC-A regulates intracellular $\mathrm{Ca}(2+)$ homeostasis in cardiomyocytes. Pflugers Arch. https://doi.org/10.1007/s00424-021-02513-6

Publisher's note Springer Nature remains neutral with regard to jurisdictional claims in published maps and institutional affiliations. 